\title{
Novel PLGA-based nanoparticles for the oral delivery of insulin
}

\author{
This article was published in the following Dove Press journal: \\ International Journal of Nanomedicine \\ 19 March 2015 \\ Number of times this article has been viewed
}

\author{
Sampath Malathi' \\ Perumal Nandhakumar ${ }^{2}$ \\ Velayudham Pandiyan ${ }^{2}$ \\ Thomas J Webster ${ }^{3}$ \\ Sengottuvelan \\ Balasubramanian' \\ 'Department of Inorganic Chemistry, \\ Guindy Campus, University of \\ Madras, Chennai, Tamil Nadu, \\ India; ${ }^{2}$ Department of Veterinary \\ Biochemistry, Madras Veterinary \\ College, Chennai, Tamil Nadu, India; \\ ${ }^{3}$ Department of Chemical Engineering, \\ Northeastern University, Boston, USA
}

Correspondence: Sengottuvelan Balasubramanian

Department of Inorganic Chemistry, Guindy Campus, University of Madras,

Chennai 600 025, Tamil Nadu, India

Tel +9l 4422202794

Fax +91 4422300488

Emailbala2010@yahoo.com
Background: Insulin is the drug therapy for patients with insulin-dependent diabetes mellitus. A number of attempts have been made in the past to overcome the problems associated with the oral delivery of insulin, but with little success. Orally administered insulin has encountered with many difficulties such as rapid degradation and poor intestinal absorption. The potential use of D- $\alpha$-tocopherol poly(ethylene glycol) 1000 succinate (TPGS)-emulsified poly(ethylene glycol) (PEG)-capped poly(lactic-co-glycolic acid) (PLGA) nanoparticles (NPs) was investigated for sustained delivery of insulin (IS).

Objective: To investigate the efficacy of TPGS-emulsified PEG-capped PLGA NPs (TPPLG NPs) as a potential drug carrier for the oral delivery of insulin.

Methods: A series of biodegradable low-molecular-weight PLGA (80/20 [PLG4] and 70/30 [PLG6]) copolymers were synthesized by melt polycondensation. The commercial insulin-loaded TPGS-emulsified PEG-capped PLGA NPs (ISTPPLG NPs) were synthesized by water-oilwater emulsion solvent evaporation method. The physical and chemical properties of PLGA copolymers, particle size, zeta potential, and morphology of the NPs were examined. The in vivo studies of ISTPPLG NPs were carried out in diabetic rats by oral administration.

Results: The maximum encapsulation efficiency of ISTPPLG6 NPs was $78.6 \% \pm 1.2 \%$, and the mean diameter of the NPs was $180 \pm 20 \mathrm{~nm}$. The serum glucose level was significantly (twofold) decreased on treatment with ISTPPLG NPs, and there was a threefold decrease with insulinloaded PLGA (70/30) NPs when compared to that of free insulin-treated diabetic rats. The results show that the oral administration of ISTPPLG6 NPs is an effective method of reducing serum glucose level for a period of 24 hours. Histopathological studies reveal that ISTPPLG NPs could restore the damage caused by streptozotocin in the liver, kidneys, and pancreas, indicating its biocompatibility and regenerative effects.

Conclusion: ISTPPLG6 NPs can act as potential drug carriers for the oral delivery of insulin.

Keywords: drug delivery, polymer nanoparticles, poly(ethylene glycol), tocopherol poly(ethylene glycol) 1000 succinate, hypoglycemic effect, histopathology

\section{Introduction}

Diabetes, a metabolic and chronic disorder, can be defined as a loss of glycemic control. Insulin is the drug therapy for patients with insulin-dependent diabetes mellitus (or type 1 diabetes), ${ }^{1}$ and it is an important hormone for the regulation of blood glucose level. Its aggregation and consequent precipitation has been a major barrier to the development of long-term delivery. ${ }^{2}$ Insulin shows poor physical and chemical stability and relatively short plasma half-life. Low $\mathrm{pH}$ and protease hydrolysis are the major limitations for intact insulin intestinal absorption and transport into systemic circulation, which is further reduced by its larger molecular weight and hydrophilic nature..$^{3-8}$ The oral administration of insulin is a preferred route for patients on chronic 
treatment because it is a safe and more convenient method. In order to improve the bioavailability of insulin, carriers such as polymeric nanoparticles (NPs), ${ }^{9}$ liposomes, ${ }^{10}$ and lipids ${ }^{11}$ have been developed. Damgé et al ${ }^{12}$ first reported in 1988 that insulin-loaded poly(isobutylcyanoacrylate) NPs had a long-term hypoglycemic effect in diabetic rats after oral administration.

Microparticles/NPs of biodegradable polymers have emerged as potential candidates for controlled and oral delivery of protein and peptide drugs. The NPs have the advantage to pass through the gastrointestinal (GI) tract and to be taken by the M cells of Peyer's patches, a type of lymphatic island within the intestinal tract that represents the major gateway through which NPs are absorbed, when compared to microparticles. ${ }^{13}$ Poly(lactic-co-glycolic acid) (PLGA) NPs have been extensively employed as a nanocarrier because of their ability to encapsulate and release drugs in a controlled manner. ${ }^{14-16}$ Moreover, their biocompatibility, biodegradability, and potential as transmucosal carrier have been reported for both nasal and oral administrations. ${ }^{17,18}$ Nevertheless, they have certain limitations such as selectivity in their interaction with mucosal surfaces. Poly(ethylene glycol) (PEG) plays an important role in improving both the stability of the colloidal systems in biological fluids and their transport across different mucosal surfaces. ${ }^{19-24}$ This improvement has been interpreted as a consequence of the stabilization of NPs and their facilitated diffusion across the mucus. ${ }^{25}$ More importantly, the main advantages of D- $\alpha$-tocopherol poly(ethylene glycol) 1000 succinate (TPGS) are its high encapsulation efficiency and high emulsification efficiency (67 times higher than that of poly(vinyl alcohol)). ${ }^{26}$

This study was aimed at investigating the effect of insulinloaded TPGS-emulsified PEG-capped PLGA NPs (ISTPPLG NPs) in an in vivo model of diabetes. The study focuses on developing biodegradable PLGA-based insulin-loaded NPs to increase the bioavailability of insulin for its hypoglycemic effects, so that the frequency of injection is minimized.

\section{Methods}

\section{Synthesis of PLGA copolymers}

PLGA was synthesized by direct melt polycondensation method. About $0.08 \mathrm{M}(80 \%)$ lactic acid (LA) (Merck, Mumbai, India) and $0.02 \mathrm{M}(20 \%)$ glycolic acid (GA) (Merck) were uniformly mixed at a feed molar ratio of PLGA 80/20 (PLG4). The monomers were directly dehydrated for 2 hours at $120^{\circ} \mathrm{C}(100 \mathrm{mmHg})$. Stannous octoate $\left(\mathrm{Sn}(\mathrm{Oct})_{2}\right)$ catalyst was prepared with a slight modification of a reported procedure in the literature. ${ }^{27} \mathrm{Sn}(\mathrm{Oct})_{2}(0.1 \mathrm{wt} \%)$ was added to this monomer mixture and then heated to $150^{\circ} \mathrm{C}$ at $30 \mathrm{mmHg}$ for 10 hours. The copolymerization was carried out at $180^{\circ} \mathrm{C}$ $(5 \mathrm{mmHg}$ ) for 6 hours. The copolymer was soluble in chloroform (Fischer Scientific, Mumbai, India) at room temperature, and it was sonicated for 10 minutes. The soluble copolymer was precipitated by the addition of ice-cold diethyl ether (Fischer Scientific). The product was dried in a vacuum desiccator and stored at $4^{\circ} \mathrm{C}$. The same procedure was also followed for the synthesis of PLGA 70/30 (PLG6) copolymer.

\section{Preparation of ISTPPLG NPs}

About $100 \mathrm{mg}$ of PLGA copolymer (PLG4:PLGA 80/20) and $50 \mathrm{mg}$ of TPGS (Sigma, Bangalore, India) were dissolved in 5 $\mathrm{mL}$ of acetone (Fischer Scientific). The solution was sonicated for 60 seconds using a probe sonicator (Hielscher sonicator, ultrasonic processor UP 100H, Berlin, Germany [100 W, 30 $\mathrm{kHz}, 0.8$ cycle, $80 \%$ amplitude]), and it was stirred continuously at $4^{\circ} \mathrm{C}$. To this, $2 \mathrm{~mL}$ of Huminsulin $(100 \mathrm{IU} / \mathrm{mL}$, EliLilly, Haryana, India) was added while stirring the solution and it was further sonicated for 60 seconds. The solution was kept in an ice bath and it formed a water-in-oil (W/O) emulsion. It was again sonicated for 180 seconds with $10 \mathrm{~mL}$ of aqueous PEG 2000 (PEG 2K; 1\% w/v, Sigma, India) solution to form a double emulsion $(\mathrm{W} / \mathrm{O} / \mathrm{W})$, and it was stirred continuously for 6 hours to remove the organic solvent. Then, it was centrifuged (Remi high-speed centrifuge C-24, Remi House, Mumbai, India) at $12,000 \mathrm{rpm}\left(4^{\circ} \mathrm{C}\right)$ for 15 minutes and the ISTPPLG4 NPs were formed (Figure 1). The residue was freeze-dried (Christ Alpha 2-4 LSC freeze dryer, Martin Christ Gefriertocknungsanlagen $\mathrm{GmbH}$, Osterode am Harz, Germany) for 12 hours, and the pellet obtained was stored at $4^{\circ} \mathrm{C}$ for further studies. The same protocol was also followed for the synthesis of copolymer PLG6 (PLGA 70/30).

\section{Determination of particle size}

The particle size and the zeta potential were determined by measuring the electrophoretic mobility of the NP with Zetatrac (Microtrac Inc., York, PA, USA). Samples were prepared by diluting with milli-Q water, and all measurements were carried out in triplicate.

\section{Transmission electron microscopy}

Transmission electron microscopic (TEM) images were obtained using the JEOL 100CXII TEM(Tokyo, Japan), $100 \mathrm{kV}$. The samples were suspended in phosphate buffer and a drop of the colloidal solution was coated on a copper grid. The samples were dried and kept under vacuum in a vacuum desiccator before placing them on the specimen holder. 


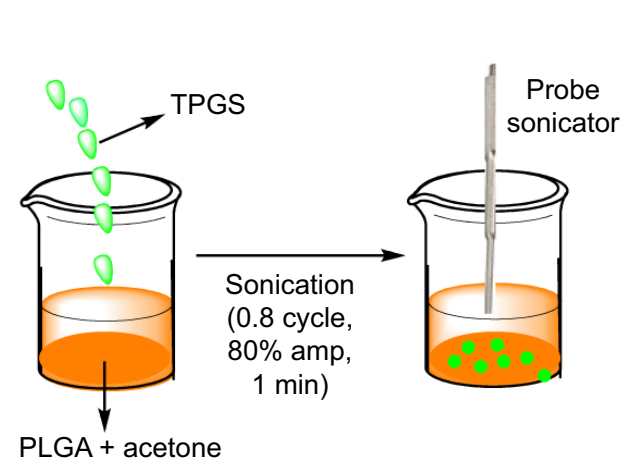

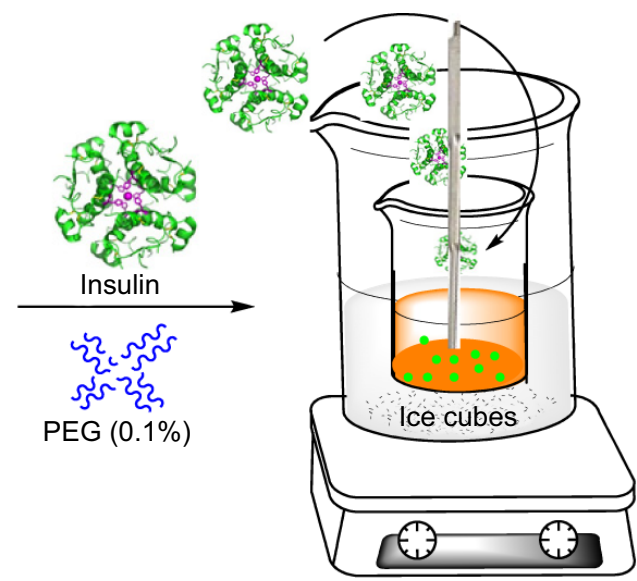

W/O/W emulsion

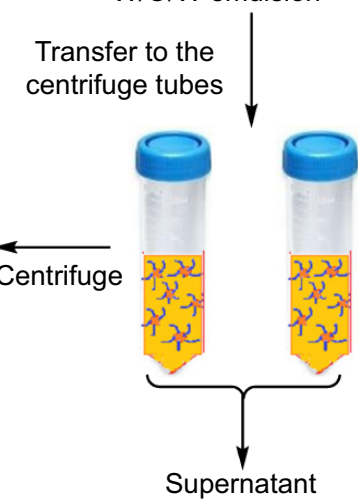

Figure I Synthesis of insulin-loaded TPGS-emulsified PEG-capped PLGA nanoparticles.

Abbreviations: PEG, poly(ethylene glycol); PLGA, poly(lactic-co-glycolic) acid; TPGS, tocopherol poly(ethylene glycol) I000 succinate.

\section{Determination of drug loading efficiency} About $5 \mathrm{mg}$ of insulin-loaded polymeric NPs (ISTPPLG4 NPs and ISTPPLG6 NPs) was dissolved in $5 \mathrm{~mL}$ of acetone using vortex for 15 minutes, and it was centrifuged at 12,000 rpm $\left(4^{\circ} \mathrm{C}\right)$ for 10 minutes. The amount of insulin present in the supernatant was measured spectrophotometrically (Perkin-Elmer, $\lambda 35$, Waltham, MA, USA) at $280 \mathrm{~nm}$.

$\begin{aligned} & \text { Drug loading } \\ & \text { efficiency }(\%)\end{aligned}=\frac{\text { Weight of insulin in polymeric nanoparticles }}{\text { Weight of insulin in formulation }} \times 100$

\section{In vivo studies}

Male rats weighing 180-250 g were randomly divided into five groups each consisting of eight animals. The animals were identified by marking at different parts of the body. The mean body weight variation did not exceed $\pm 20 \%$. The animals were maintained on standard rat feed supplied by M/s. Tetragon Chemie Private Limited, Bangalore, India. All animals were housed in cages with 12/12 hours light/dark cycle. The animals were provided feed and water ad libitum throughout the experimental period. The animals were acclimatized for 1 week prior to the start of the experiment. The animal experiments were carried out after prior approval from the Institutional Animal Ethical Committee (IAEC), MVC, Chennai, India. The blood glucose level was assessed to rule out spontaneous diabetes in the rats before administration of streptozotocin (STZ) (Sigma Chemicals Co., St Louis, MO, USA) injection. Those animals showing normal blood glucose levels of $50-70 \mathrm{mg} / \mathrm{dL}$ were selected for the study.

\section{Induction of diabetes mellitus}

The animals were fasted overnight and a single intraperitoneal injection of a freshly prepared solution of STZ $(45 \mathrm{mg} / \mathrm{kg}$ body weight) in $0.1 \mathrm{M}$ cold citrate buffer ( $\mathrm{pH} 4.5$ ) was given to induce diabetes. The animals were allowed access to $5 \%$ glucose solution overnight, to prevent total hypoglycemia induced by STZ by massive pancreatic insulin release. The animals were considered as diabetic if their blood glucose values were above $250 \mathrm{mg} / \mathrm{dL}$ on the third day ( 72 hours) after STZ injection. The treatment was started on the fourth day after STZ injection and that day was considered as the first day of treatment. 


\section{Experimental design}

The animals were divided into five groups. Group 1 served as normal control. Group 2 rats were diabetic control without any treatment. Group 3 rats were treated with Huminsulin at a dose of $20 \mathrm{IU} / \mathrm{kg}$ bodyweight. Group 4 rats were treated with ISTPPLG4 NPs at a predetermined dose of $20 \mathrm{IU} / \mathrm{kg}$ bodyweight. Group 5 rats were treated with ISTPPLG6 NPs at a predetermined dose of $20 \mathrm{IU} / \mathrm{kg}$ bodyweight.

\section{Biochemical assays}

Blood samples for biochemical analysis were collected at regular intervals during the experiment in a vacutainer with coagulant activators, and the serum was separated by centrifugation at 3,000 rpm for 15 minutes, for the analysis of glucose, insulin (IRMA kit, Board of Radiation and Isotope Technology, Mumbai, India), cholesterol, high-density lipoprotein cholesterol, triacylglycerol, total protein, urea, creatinine, aspartate aminotransferase (ALT), and alanine aminotransferase (AST) as per the manufacturer's protocol described in the standard kits (Agappe Diagnostics Ltd., Ernakulam, Kerala, India), using CECIL CE 2021 UV spectrophotometer (Cecil, Cambridge, UK).

\section{Histopathology}

The collected tissues were embedded in paraffin after fixing the tissues in $10 \%$ formalin. Serial sections were cut and stained with hematoxylin and eosin. The sections were examined under high-power microscope $(200 \times)$ and photomicrographs were taken.

\section{Statistical analysis}

The results were expressed as mean \pm standard error. All the data were analyzed by one-way analysis of variance (ANOVA), followed by Duncan's multiple range test. ${ }^{28}$ A value of $P<0.05$ was considered statistically significant.

\section{Results}

The biocompatible low-molecular-weight PLGA copolymers were synthesized by melt polycondensation of the monomer lactic acid and GA using $\mathrm{Sn}(\mathrm{Oct})_{2}$ as the catalyst. The polymer composition and molecular weight were obtained by nuclear magnetic resonance and gel permeation chromatography techniques. The calculated copolymer ratio from nuclear magnetic resonance is $78 / 22$ (PLG4) and 68/32 (PLG6) (Table 1). The average molecular weight (Mw) of PLG4 and PLG6 copolymers was found to be 15,400 and 11,000 Da, respectively (Table 1).

The mean diameter of ISTPPLG4 NPs and ISTPPLG6 NPs was found to be 180 and $120 \mathrm{~nm}$, respectively, by the dynamic light scattering (DLS) method (Table 1). The ISTPPLG4 NPs exhibit a larger average diameter when compared to that of ISTPPLG6 $\mathrm{NPs}$ (Figure 2). The zeta potential of ISTPPLG4 NPs is $-2.90 \mathrm{mV}$, whereas that of ISTPPLG6 NPs is $-6.59 \mathrm{mV}$. TEM photomicrographs (Figure 3) show that ISTPPLG NPs have a smooth spherical surface and relatively uniform size distribution. The size of the NPs is below $200 \mathrm{~nm}$, which is further confirmed by particle size analysis. The loading efficiency of ISTPPLG4 and ISTPPLG6 NPs is $62.3 \% \pm 1.1 \%$ and $78.6 \% \pm 1.2 \%$, respectively.

\section{In vitro release study of ISTPPLG NPs}

The in vitro release profile of the insulin-loaded ISTPPLG NPs was observed at $37^{\circ} \mathrm{C}$ in phosphate-buffered saline medium. The quantity of insulin released was followed spectrophotometrically at $280 \mathrm{~nm}$. The release of insulin was observed in both the drug-loaded polymeric NPs (ISTPPLG4 NPs and ISTPPLG6 NPs) up to 36 hours (Figure 4).

\section{Effect of ISTPPLG NPs on serum glucose and insulin levels in STZ-induced diabetic rats}

The hypoglycemic effect of orally delivered insulin-loaded polymeric NPs (ISTPPLG NPs) was evaluated at $20 \mathrm{IU} / \mathrm{kg}$ dose in diabetic rats. The schematic representation of insulin released from the ISTPPLG NPs at different time intervals is shown in Figure 5. Blood glucose and serum insulin levels were compared to those of orally delivered

Table I Characteristics of PLGA copolymer and their insulin-loaded nanoparticles

\begin{tabular}{|c|c|c|c|c|c|c|}
\hline \multicolumn{3}{|c|}{ PLGA polymer } & \multicolumn{4}{|c|}{ Nanoparticles } \\
\hline $\begin{array}{l}\text { Sample } \\
\text { code }\end{array}$ & $\begin{array}{l}\text { Polymer } \\
\text { ratio }^{\mathrm{a}}\end{array}$ & $\begin{array}{l}\text { Molecular } \\
\text { weight }^{\mathrm{b}}(\mathrm{Da})\end{array}$ & $\begin{array}{l}\text { Sample } \\
\text { code }\end{array}$ & $\begin{array}{l}\text { Particle } \\
\text { size }(\mathrm{nm})\end{array}$ & $\begin{array}{l}\text { Zeta potential } \\
(\mathrm{mV})\end{array}$ & $\begin{array}{l}\text { Drug loading } \\
\text { efficiency (\%) }\end{array}$ \\
\hline PLG4 & $78 / 22$ & 15,400 & ISTPPLG4 & $180 \pm 20$ & $-2.90 \pm 0.3$ & $62.3 \pm 1.1$ \\
\hline PLG6 & $68 / 32$ & 11,000 & ISTPPLG6 & $120 \pm 20$ & $-6.59 \pm 0.2$ & $78.6 \pm 1.2$ \\
\hline
\end{tabular}

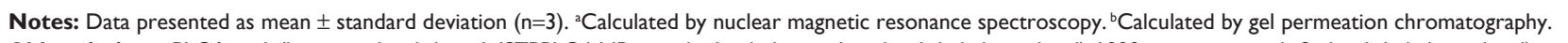
Abbreviations: PLGA, poly(lactic-co-glycolic) acid; ISTPPLG4 NPs, insulin-loaded tocopherol poly(ethylene glycol) 1000 succinate-emulsified poly(ethylene glycol)-capped poly(lactic-co-glycolic acid) (78/22) nanoparticles; ISTPPLG6 NPs, insulin-loaded tocopherol poly(ethylene glycol) 1000 succinate-emulsified poly(ethylene glycol)-capped poly(lactic-co-glycolic acid) (68/32) nanoparticles. 

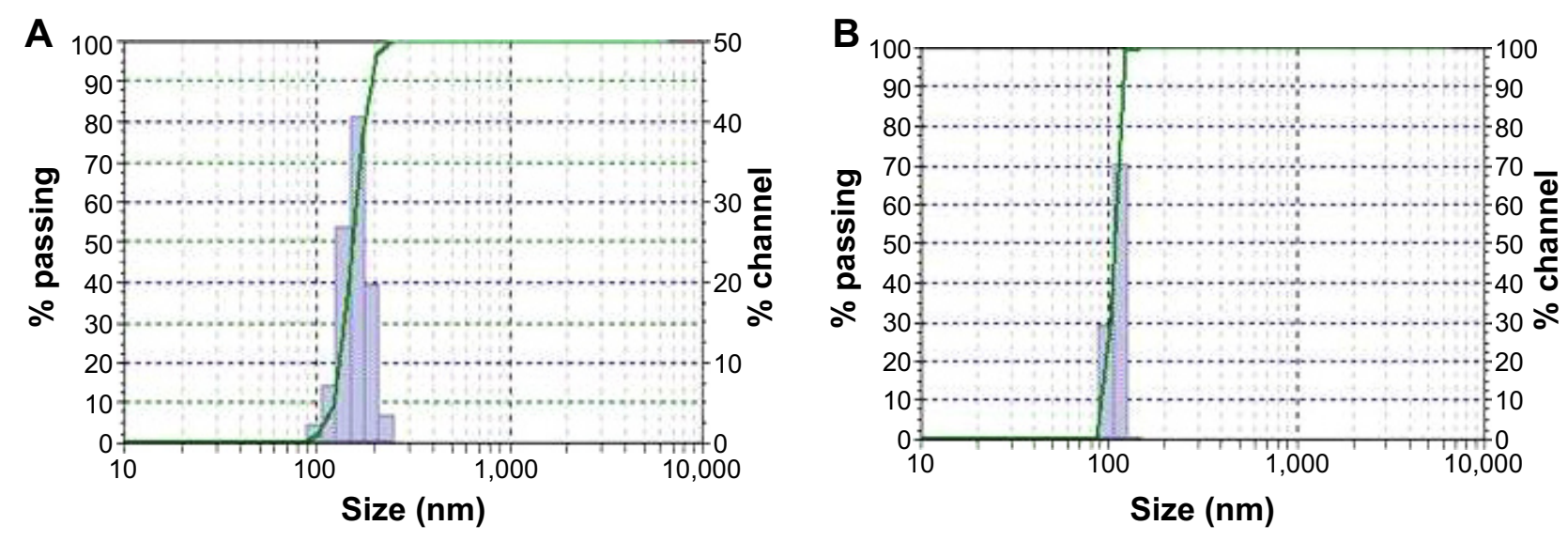

Figure 2 Particle size analysis of (A) ISTPPLG4 NPs and (B) ISTPPLG6 NPs.

Abbreviations: ISTPPLG4 NPs, insulin-loaded tocopherol poly(ethylene glycol) 1000 succinate-emulsified poly(ethylene glycol)-capped poly(lactic-co-glycolic acid) (78/22) nanoparticles; ISTPPLG6 NPs, insulin-loaded tocopherol poly(ethylene glycol) 1000 succinate-emulsified poly(ethylene glycol)-capped poly(lactic-co-glycolic acid) (68/32) nanoparticles.

free form of insulin at the same dose in diabetic rats. The changes observed in the blood glucose level versus time profiles are reproduced in Figure 6A. The blood glucose level was suppressed during the oral administration of ISTPPLG NPs when compared to that of diabetic control. The free insulin maintains the glucose level up to 3 hours and increases rapidly and then returns to the original level. More interestingly, the blood glucose concentration undergoes a maximum decrease at the 24th hour $(P<0.05)$ for ISTPPLG6 NPs and at the 6th hour $(P<0.05)$ for ISTPPLG4 NPs. Following this, the blood glucose concentration increases rapidly and returns to the original level after 12th and 24th hours, respectively, for ISTPPLG4 and ISTPPLG6 NPs. The results indicate that insulin absorption through the intestinal membrane was enhanced by the encapsulation of insulin in TPPLG NPs. The decrease in glucose levels was significantly higher in diabetic rats that received insulin-encapsulated NPs than in rats that received free insulin. The absorption of insulin was investigated by measuring the serum insulin concentration. The changes in serum insulin level with respect to time in the diabetic rats are shown in Figure 6B. The serum insulin concentration increases dramatically after the administration of ISTPPLG NPs. The ISTPPLG6 NPs-administered rats exhibit a maximum insulin value close to $6 \mu \mathrm{IU} / \mathrm{dL}$ within 12 hours $\left(t_{\max }\right)$, which is significantly higher when compared to that of other groups. However, ISTPPLG4 NPs-administered rats show a maximum insulin value close to $5 \mu \mathrm{IU} / \mathrm{dL}$ within 6 hours $\left(t_{\max }\right)$, which is significantly higher than that of the free insulin group.
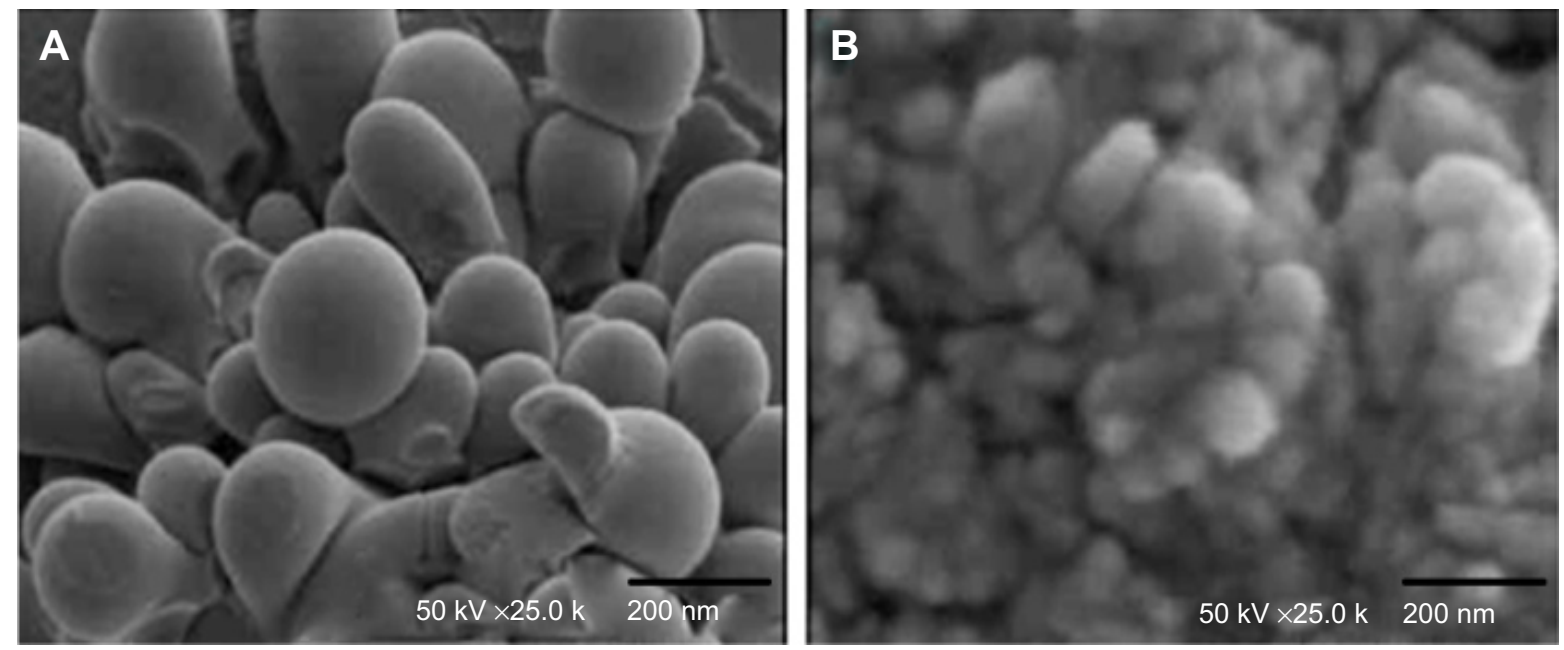

Figure 3 TEM micrographs of (A) ISTPPLG4 NPs and (B) ISTPPLG6 NPs.

Abbreviations: ISTPPLG4 NPs, insulin-loaded tocopherol poly(ethylene glycol) 1000 succinate-emulsified poly(ethylene glycol)-capped poly(lactic-co-glycolic acid) (78/22) nanoparticles; ISTPPLG6 NPs, insulin-loaded tocopherol poly(ethylene glycol) 1000 succinate-emulsified poly(ethylene glycol)-capped poly(lactic-co-glycolic acid) (68/32) nanoparticles; TEM, transmission electron microscope. 


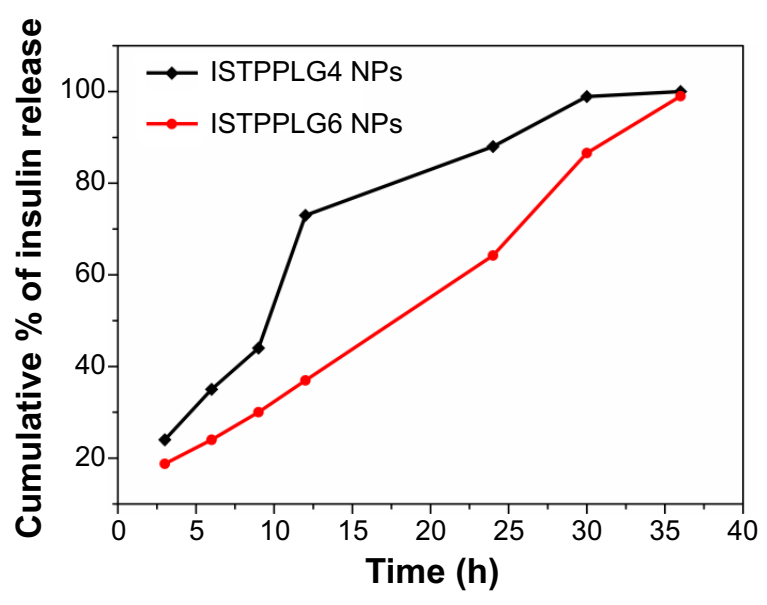

Figure 4 In vitro release profile of ISTPPLG NPs.

Abbreviation: ISTPPLG NPs, insulin-loaded tocopherol poly(ethylene glycol) 1000 succinate-emulsified poly(ethylene glycol)-capped poly(lactic-co-glycolicacid) nanoparticles; ISTPPLG4 NPs, insulin-loaded tocopherol poly(ethylene glycol) 1000 succinate emulsified poly(ethylene glycol)-capped poly(lactic-co-glycolic acid) 78/22 nanoparticles; ISTPPLG6 NPs, insulin-loaded tocopherol poly(ethylene glycol) 1000 succinate emulsified poly(ethylene glycol)-capped poly(lactic-co-glycolic acid) 68/32 nanoparticles.

\section{Effect of ISTPPLG NPs on lipid profiles and status of biochemical markers (urea, creatinine, AST, and ALT) in STZ-induced diabetic rats}

The serum triacylglycerol and serum cholesterol levels were significantly reduced on treatment with ISTPPLG4 and ISTPPLG6 NPs when compared to those of diabetic rats and insulin-treated diabetic rats at the 24th hour. The levels of serum urea, creatinine, AST, and ALT were significantly reduced on treatment with ISTPPLG4 and ISTPPLG6 NPs when compared to those of diabetic and insulin-treated diabetic rats at the 24th hour (Table 2).

\section{Effect of ISTPPLG NPs on} histopathological changes of liver, kidney, and pancreas in STZ-induced diabetic rats

The histopathological studies were carried out 12 hours after the oral administration of ISTPPLG NPs. Liver sections of diabetic control rats showed marked microscopical changes such as hydropic degeneration of hepatocytes and dilatation of sinusoids when compared to that of normal control rats. The treatment with ISTPPLG6 NPs diminished the intensity of degeneration and dilatation of sinusoids. The microscopic examination of diabetic control rats (kidney) showed marked changes such as tubular epithelial cell degeneration and cystic dilatation of tubules. The treatment with ISTPPLG6 NPs reduced the intensity of degeneration and cystic dilatation of tubules. The pancreatic sections of diabetic control and insulin-treated and ISTPPLG6 NPstreated diabetic groups showed marked microscopical changes such as atrophy and reduction in the number of islet cells (Figure 7).

\section{Discussion}

The low-molecular-weight PLG4 (15,400 Da) and PLG6 $(11,000 \mathrm{Da})$ were synthesized by melt polycondensation method, and their molecular weights were determined by gel

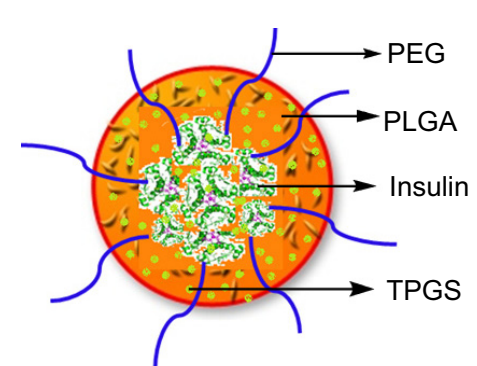

ISTPPLG NPS
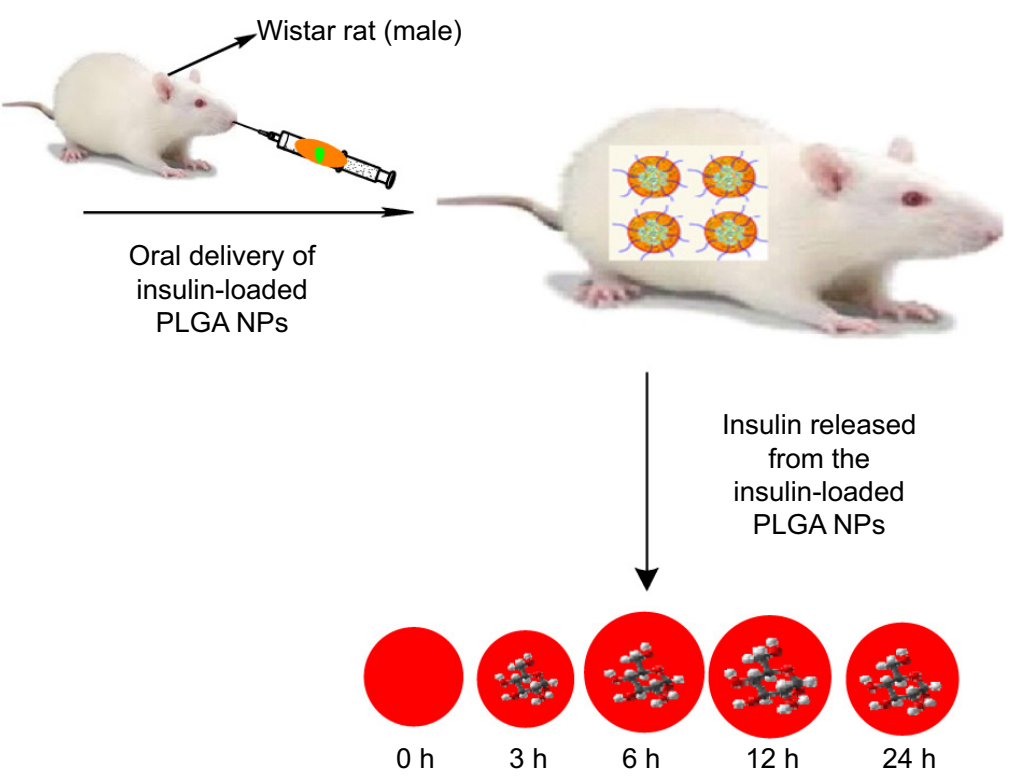

Serum blood glucose levels at different time intervals

Figure 5 Schematic illustration of insulin release from insulin-loaded TPGS-emulsified PEG-capped PLGA nanoparticles.

Abbreviations: PEG, poly(ethylene glycol); PLGA, poly(lactic-co-glycolic) acid; TPGS, tocopherol poly(ethylene glycol) 1000 succinate. 
A

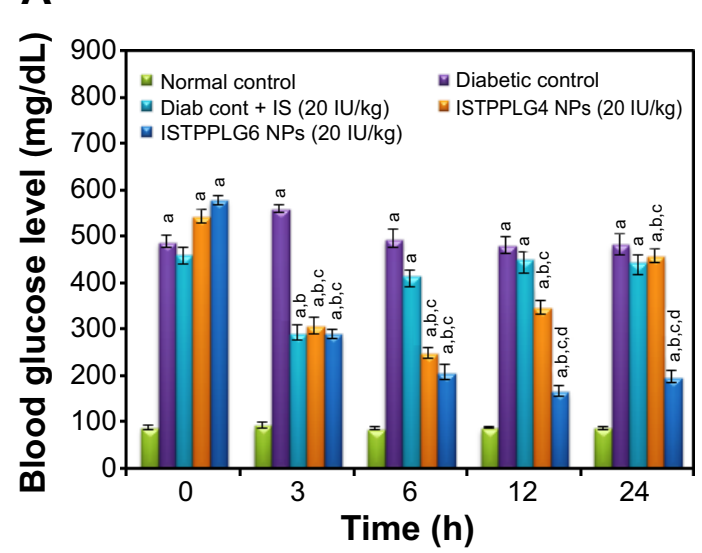

B

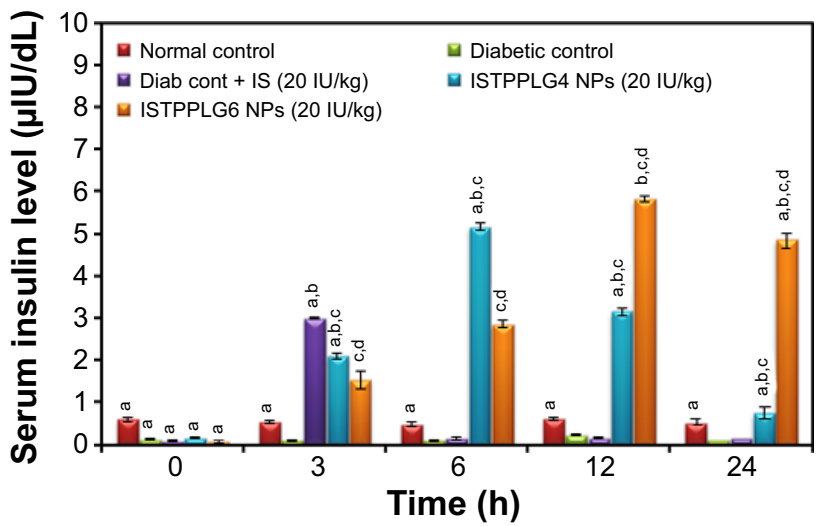

Figure 6 Effect of ISTPPLG NPs on serum glucose and insulin levels in STZ-induced diabetic rats. (A) Variation of blood glucose level with time (20 IU/kg). (B) Variation of serum insulin level with time $(20 \mathrm{IU} / \mathrm{kg})$.

Note: Data show the mean \pm SD ( $n=8)$.

Abbreviations: Cont, control; IS, insulin; ISTPPLG4 NPs, insulin-loaded tocopherol poly(ethylene glycol) I000 succinate-emulsified poly(ethylene glycol)-capped poly(lacticco-glycolic acid) (78/22) nanoparticles; ISTPPLG6 NPs, insulin-loaded tocopherol poly(ethylene glycol) 1000 succinate-emulsified poly(ethylene glycol)-capped poly(lactic-coglycolic acid) (68/32) nanoparticles; SD, standard deviation; Diab cont, diabetic control.

permeation chromatography. The PLGA copolymers have been extensively employed as a carrier for oral delivery of insulin. ${ }^{29}$ In this study, the NPs have been synthesized by emulsion-solvent evaporation method. In this formulation, an aqueous insulin solution is emulsified into an organic solvent such as methylene chloride containing PLGA to form a W/O emulsion. The formation of a primary W/O emulsion is considered as the main cause for protein inactivation and aggregation. ${ }^{30}$ PEG has been employed as a capping agent to avoid these problems. PEG interacts with insulin via a hydrophobic interaction while, on the other hand, PEG establishes a large number of hydrogen bonds with water. The unique nature of PEG enhances the stability of the protein and maintains an aqueous layer that retains the water molecules necessary for the biological function of the protein. TPGS is a good emulsifier and enhances the solubility and bioavailability. The mean diameter of the particle size is reduced significantly from 180 to $120 \mathrm{~nm}$ when the content of GA in the copolymers was increased from $20 \%$ to $30 \%$. The experimental conditions such as sonication time, sonication frequency, centrifugation time, and speed have been optimized in such a way to get almost similar-sized NPs in repeated trials. The particle diameter $<500 \mathrm{~nm}$ is desirable to increase the contact with the intestinal mucosa and to facilitate insulin permeation through the intestinal tract. ${ }^{31}$ In this study, zeta potential values indicate that the stability of the NPs stored in lyophilized state is more than that of the colloidal form, suggesting that the particles should be stored in a lyophilized state. Abdelwahed et $\mathrm{al}^{32}$ have reported that NP formulations stored in a colloidal state cause more stability problems than those stored in the dry form. However, the insulin loading efficiency also decreases from $78 \%$ to $64 \%$. It is known that when the hydrophilic content (GA) increases, the hydrophilic drug (insulin) loading efficiency also increases. The TEM study shows the spherical nature of the particles with an average size of $<200 \mathrm{~nm}$.

Table 2 Biochemical parameters of ITPPLG NPs

\begin{tabular}{llllllll}
\hline Groups & $\begin{array}{l}\text { Total protein } \\
(\mathbf{g} / \mathbf{d L})\end{array}$ & $\begin{array}{l}\text { Triacyl glycerol } \\
(\mathbf{m g} / \mathbf{d L})\end{array}$ & $\begin{array}{l}\text { Cholesterol } \\
(\mathbf{m g} / \mathbf{d L})\end{array}$ & $\begin{array}{l}\text { Urea } \\
(\mathbf{m g} / \mathbf{d L})\end{array}$ & $\begin{array}{l}\text { Creatinine } \\
(\mathbf{m g} / \mathbf{d L})\end{array}$ & $\begin{array}{l}\text { AST } \\
(\mathbf{U} / \mathbf{L})\end{array}$ & $\begin{array}{l}\text { ALT } \\
(\mathbf{U} / \mathbf{L})\end{array}$ \\
\hline Normal control & $7.29 \pm 0.23$ & $122.58 \pm 18.97$ & $69.77 \pm 2.91$ & $37.88 \pm 2.22$ & $0.62 \pm 0.03$ & $60.61 \pm 3.98$ & $23.61 \pm 1.48$ \\
Diabetic control & $6.43 \pm 0.08^{\mathrm{a}}$ & $513.10 \pm 44.3^{\mathrm{a}}$ & $110.69 \pm 1.56^{\mathrm{a}}$ & $61.37 \pm 2.09^{\mathrm{a}}$ & $2.54 \pm 0.05^{\mathrm{a}}$ & $170 \pm 2.37^{\mathrm{a}}$ & $74.29 \pm 1.57^{\mathrm{a}}$ \\
Diabetic control + insulin & $6.61 \pm 0.06^{\mathrm{b}}$ & $501.94 \pm 23.39^{\mathrm{a}}$ & $108.23 \pm 1.47^{\mathrm{a}, \mathrm{b}}$ & $62.06 \pm 1.72^{\mathrm{a}}$ & $2.39 \pm 0.05^{\mathrm{a}}$ & $164.41 \pm 4.20^{\mathrm{a}}$ & $75.01 \pm 1.73^{\mathrm{a}}$ \\
ISTPPLG4 NPs & $6.87 \pm 0.04$ & $300.67 \pm 21.05^{\mathrm{b}, \mathrm{c}}$ & $88.18 \pm 1.98^{\mathrm{b}, \mathrm{c}}$ & $55.8 \pm 1.55$ & $1.69 \pm 0.10^{\mathrm{b}, \mathrm{c}}$ & $135.81 \pm 8.42$ & $59.37 \pm 3.97$ \\
ISTPPLG6 NPs & $7.05 \pm 0.10$ & $286.69 \pm 17.70^{\mathrm{a}-\mathrm{c}}$ & $76.84 \pm 1.00^{\mathrm{a}-\mathrm{c}}$ & $42.19 \pm 1.34^{\mathrm{a}-\mathrm{d}}$ & $1.04 \pm 0.14$ & $103.87 \pm 6.37$ & $46.26 \pm 2.29$ \\
\hline
\end{tabular}

Notes: Data presented as mean \pm standard deviation $(n=8)$. Significance level, $P<0.05$. aSignificant compared with control. ${ }^{b}$ Significant compared with diabetic control. 'Significant compared with insulin-treated diabetic rats. 'Significant compared with ISTPPLG4 NPs.

Abbreviations: ISTPPLG4 NPs, insulin-loaded tocopherol poly(ethylene glycol) 1000 succinate-emulsified poly(ethylene glycol)-capped poly(lactic-co-glycolic acid) (78/22) nanoparticles; ISTPPLG6 NPs, insulin-loaded tocopherol poly(ethylene glycol) 1000 succinate-emulsified poly(ethylene glycol)-capped poly(lactic-co-glycolic acid) (68/32) nanoparticles; AST, aspartate aminotransferase; ALT, alanine aminotransferase. 

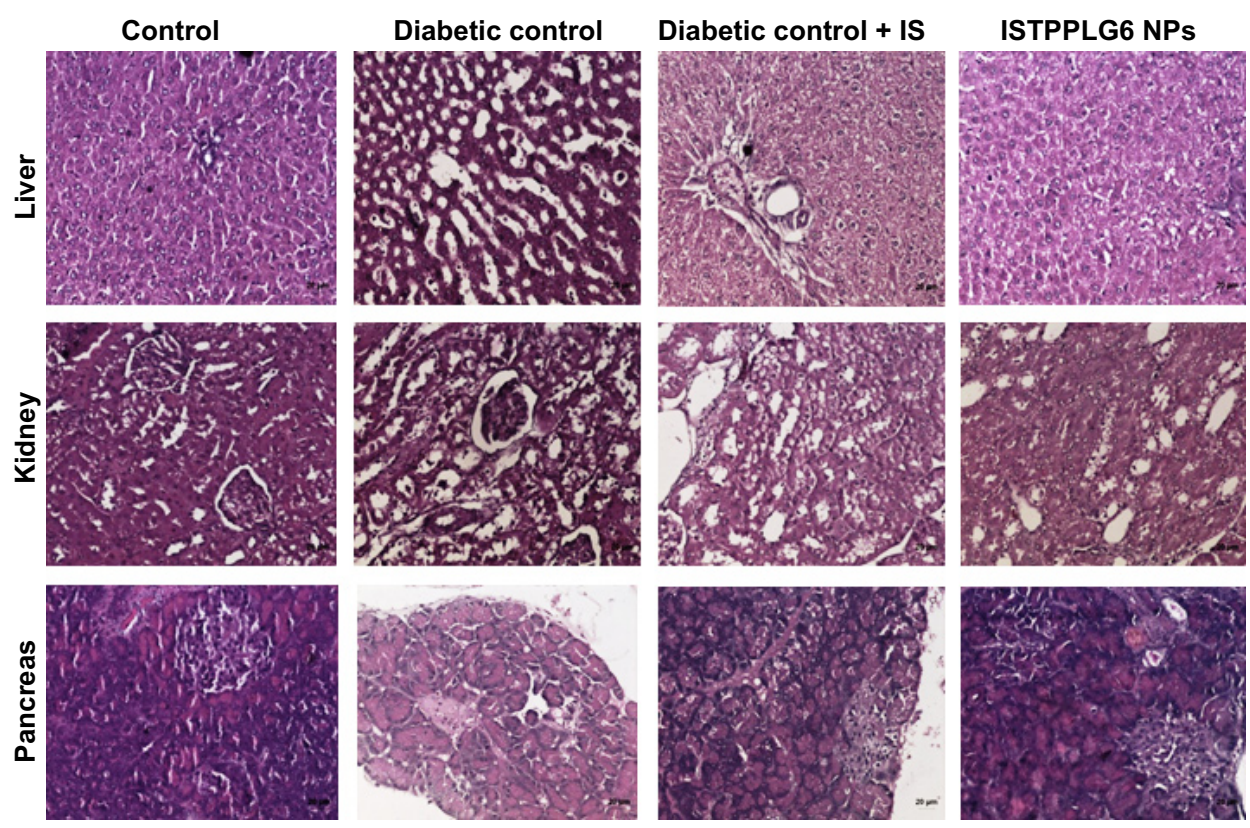

Figure 7 Effect of ISTPPLG6 NPs on histopathological changes of the liver, kidney, and pancreas.

Abbreviation: ISTPPLG6 NPs, insulin-loaded tocopherol poly(ethylene glycol) 1000 succinate-emulsified poly(ethylene glycol)-capped poly(lactic-co-glycolic acid) (68/32) nanoparticles.

\section{Insulin hypoglycemic effect}

The schematic illustration of the oral administration of insulin-loaded polymeric NPs (ISTPPLG NPs) and the release of insulin from the ISTPPLG NPs is shown in Figure 5. Insulin, when administered orally, undergoes degradation in the stomach due to gastric enzymes. ${ }^{33}$ Therefore, insulin should be enveloped in a matrix-like system to protect it from gastric enzymes. This can be achieved by encapsulating the insulin molecules in ISTPPLG matrix. The PEG coating protects the insulin from the gastric enzymes. Due to the mucoadhesive property and solubility of PEG, and its capability to prevent protein aggregation, the formulation remained concentrated in the small intestine for a longer period, resulting in slower absorption and longer availability in the bloodstream. PEG serves as a nanosphere stabilizer to improve the half-life of the insulin and increases the residence time along the intestine. Tobio et $\mathrm{a}^{34}$ have reported that coating $\sim 160 \mathrm{~nm}$ PLA NPs with PEG imparts additional protection against enzyme-induced aggregation and degradation in simulated GI fluids in vitro.

The increase in the insulin loading efficiency could be due to the decrease in particle size and the increase of the hydrophilicity of the polymeric NPs with higher TPGS content. Ruan et $\mathrm{al}^{35}$ have described that the involvement of either low (2\%) or high (10\%) concentrations of TPGS as additive in fabricating HAS-loaded PLGA and PELA NPs had decreased the encapsulation efficiency around $25 \%$, compared to that of NPs without any additives. Insulin is physically and uniformly dispersed in the TPPLG matrix, and these NPs degrade into biologically acceptable compounds by hydrolysis, thus delivering the insulin to the target site. The bulk erosion process occurs in the PLGA matrix where the release rate is controlled by the surface area. The polymer is degraded into lactic acids and GAs, which are eventually reduced to carbon dioxide and water by Krebs cycle. The released insulin exhibits prolonged hypoglycemic effect. These approaches substantiate the potential use of TPPLG NPs in oral administration of insulin, thereby bypassing the enzymatic degradation in the stomach.

The hypoglycemic effect of oral administration of ISTPPLG NPs was able to maintain the glucose level over a longer time than with the free insulin. Improved pharmacological effect of insulin-loaded NPs, compared to that of oral administration of free insulin, indicated that the NPs facilitated insulin absorption by potentially increasing the residence time of insulin at the intestinal mucosa and internalization through the enterocytes. ${ }^{36}$ In general, the internalization of $<500 \mathrm{~nm}$ particles in the intestinal cells and the uptake by Peyer's patches of particles $<1 \mu \mathrm{m}$ indicate that the absorption of insulin can be enhanced by the oral route. The efficacy of ISTPPLG NPs in reducing the blood glucose levels in diabetic rats in the present investigation is comparable to the hypoglycemic effect observed during 
the oral delivery of insulin using PLGA-Hp55 (hypromellose phthalate 55) NPs, ${ }^{37}$ chitosan-coated PLGA NPs, ${ }^{38}$ and sodium deoxycholate complex-loaded PLGA NPs. ${ }^{39}$ The ISTPPLG NPs were found to be effective in lowering the blood glucose level at the dose of $20 \mathrm{IU} / \mathrm{kg}$ (Figure 6A). The hypoglycemic effect appeared to be maximum in the 6th hour for ISTPPLG4 NPs, but a controlled maximum level was observed in the 12-24th hour for ISTPPLG6 NPs. In the present investigation, ISTPPLG6 NPs showed a more significant hypoglycemic effect when compared to that of ISTPPLG4 NPs.

The serum insulin level was significantly (twofold) increased on treatment with ISTPPLG4 NPs, and there was a threefold increase with ISTPPLG6 NPs when compared to that in free insulin-treated diabetic rats. The ISTPPLG6 NPs have a higher GA content when compared to that of ISTPPG4 NPs and hence exhibit a higher loading efficiency. The reason for the effective and prolonged hypoglycemic effect of ISTPPLG6 NPs is their ability to protect insulin from the enzymatic degradation in the GI tract and their particle size $(120 \pm 20 \mathrm{~nm})$, which is favorable for the insulin absorption in the intestine. The presence of PEG on the surface of ISTPPLG NPs could impart high permeation characteristic to the cell membrane of the intestine due to its amphiphilic property. Wu et $\mathrm{al}^{4}$ have described that Hp55 (hydroxypropyl methylcellulose phthalate)-coated capsule containing insulin-loaded PLGA/RS (Eudragit ${ }^{\circledR}$ RS, Evonik Industries, Essen, Germany) NPs when administered orally showed a prolonged hypoglycemic effect in STZ-induced diabetic rat model. Similarly, Kumar et $\mathrm{al}^{40}$ have investigated the use of insulin-loaded PLGA NPs for oral administration in the treatment of diabetes mellitus and found that the formulation was effective in reducing the blood glucose level in STZinduced diabetic rats. The in vitro release was also observed in a controlled manner (Figure 4) in both the cases, while in the former (ISTPPLG4 NPs) the release rate was faster when compared to that in the latter (ISTPPLG6 NPs). The insulin release was completed in 30 hours in the case of ISTPPLG4 NPs, while it took 36 hours for ISTPPLG6 NPs. A similar trend was also observed in in vivo studies.

\section{Effect of ISTPPLG NPs on lipid profiles and status of biochemical markers in diabetic rats}

A significant increase in serum triacylglycerol and total cholesterol levels was observed in diabetic rats, which is consistent with the earlier studies. ${ }^{41}$ The administration of
ISTPPLG NPs significantly reduced the serum triacylglycerol and total cholesterol levels at the 24th hour, which indicates the antihyperlipidemic effect of insulin. The administration of ISTPPLG NPs to diabetic rats resulted in the reduction of cholesterol and triacylglycerol levels at the 24th hour. This may be attributed to the increased glucose utilization and decreased mobilization of fatty acid from fat depots, which corrects the hypertriacylglycerol and cholesterol levels.

The increase in serum urea concentration in diabetic rats may be due to the depletion of serum protein, increase in the rate of circulating amino acids, and deamination in the liver that eventually elevate urea levels. It may also be due to renal dysfunction. ${ }^{42}$ The administration of ISTPPLG NPs was found to decrease the serum urea level. Creatinine is formed from creatine phosphate by nonenzymatic degradation in the muscle tissue. The alteration in the creatinine levels is taken as the indication of abnormal glomerular functions, and these changes are correlated with the nephrotoxic effects of diabetes. ${ }^{43}$

The ALT activity was increased by threefold in untreated diabetic rats when compared to that in normal rats. The aminotransferases such as ALT and AST are intracellular enzymes which have leaked into the circulation and serve as a molecular marker of tissue injury, particularly hepatocytes. During diabetes, the activities of the aminotransferases are elevated. ${ }^{44}$ The administration of ISTPPLG NPs produced a significant decrease in serum ALT activity, which was comparable to that of normal control.

\section{Effect of ISTPPLG NPs on histopathological changes of liver, kidney, and pancreas in STZ-induced diabetic rats}

The treatment with ISTPPLG NPs diminished the intensity of degeneration and dilatation of sinusoids, which indicates that ISTPPLG NPs reduce the damage in the liver tissue of diabetic rats. The kidney of diabetic control rats showed marked changes such as tubular epithelial cell degeneration and cystic dilatation of tubules, which are suggestive of diabetic nephropathy because of altered lipid, protein, and carbohydrate metabolisms. These findings are in accordance with the results of Tedong et al. ${ }^{45}$

The treatment of ISTPPLG NPs reduced the intensity of degeneration and cystic dilatation of tubules, thereby slowing down the rate of degeneration due to diabetic complication. The pancreatic sections of diabetic and ISTPPLG NPs-treated rats showed atrophy of the islets of Langerhans because of the interaction of STZ on the islet cells, which is similar to the findings of Bolkent et al. ${ }^{46}$ 


\section{Mechanism of insulin-loaded polymeric NPs transport across the cells}

The absorption of orally administered ISTPPLG NPs depends on the successful passage of the NPs through several barriers to drug delivery. The NPs can pass either between or through the cells, depending on their physicochemical properties. The mechanisms proposed for the transport of NPs through the intestinal epithelium are 1) paracellular transport, 2) passive transcytosis or receptor-mediated transcytosis, 3) transport via $\mathrm{M}$ cells of Peyer's patches, and 4) endocytosis by enterocytes. ${ }^{47}$ The larger-sized polymeric NPs $(>5 \mathrm{~nm})$ may not pass through the tight junction. In order to overcome this problem, enhancers such as PEG and TPGS which reversibly open the tight junction are incorporated in the insulin-encapsulated PLGA. The larger-sized particles can also undergo degradation, resulting in the release of insulin while they pass through transcellular as well as paracellular pathways (Figure 8).

\section{Conclusion}

In this study, low-molecular-weight PLGA copolymers with PEG as a capping agent and TPGS as an emulsifier were synthesized and employed as a carrier for insulin drug delivery. ISTPPLG NPs were prepared for the first time by the emulsion-solvent evaporation method. The NPs with the size of $<200 \mathrm{~nm}$ are spherical in nature and exhibit a loading efficiency of $\leq 78 \%$. PLGA NPs emulsified with TPGS and capped with PEG prevent enzymeinduced aggregation and degradation in simulated GI fluids in vitro and also increase the loading efficiency of insulin.
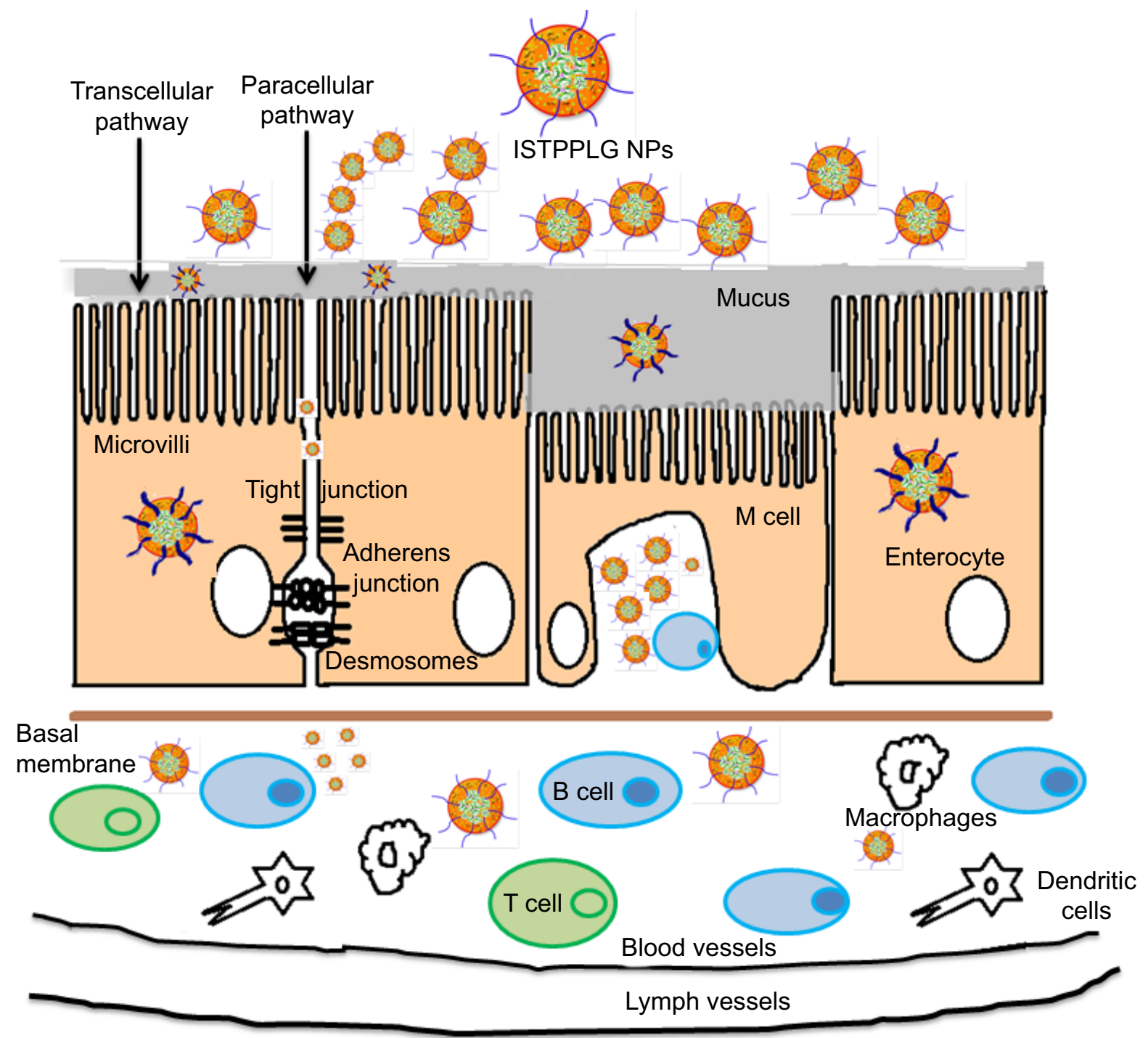

Figure 8 Schematic representation of the transport of drug-loaded polymeric nanoparticles (ISTPPLG NPs) across the intestinal epithelium.

Abbreviations: B cell, B lymphocyte; ISTPPLG NPs, insulin-loaded tocopherol poly(ethylene glycol) 1000 succinate-emulsified poly(ethylene glycol)-capped poly(lactic-co-glycolic acid) nanoparticles; $M$ cell, microfold cells; $T$ cell, $T$ lymphocyte. 
Most importantly, the hypoglycemic effect of ISTPPLG NPs was observed over a period of 24 hours. The oral administration of ISTPPLG6 NPs exhibits a maximum reduction in the blood glucose level up to 12 hours when compared to that with ISTPPLG4 NPs and control. The effect of ISTPPLG NPs on lipids and biochemical markers in diabetic rats shows a significant reduction in the cholesterol, urea, creatinine, and ALT levels. Histopathological results show that ISTPPLG NPs have a regenerative effect on diabetic rats in the liver, kidneys, and pancreas when compared to normal control. In conclusion, the ISTPPLG6 NPs are found to be potential candidates as a nanocarrier for the oral delivery of insulin.

\section{Acknowledgments}

We thank the National Center for Nanoscience and Nanotechnology (NCNST), University of Madras, Chennai, India, for TEM studies. The financial support by the Indian Council of Medical Research (ICMR), New Delhi, is gratefully acknowledged. The authors thank the Tamil Nadu Veterinary and Animal Sciences University for providing financial support for carrying out the animal experiments and Prof Thomas Webster, Northeastern University, for the support.

\section{Disclosure}

The authors report no conflicts of interest in this work.

\section{References}

1. Chien YW. Human insulin: basic sciences to therapeutic uses. Drug Dev Ind Pharm. 1996;22:753-789.

2. Brange J, Langkjaer L. Insulin structure and stability. In: Wang YJ, Pearlman R, editors. Stability and Characterization of Protein and Peptide Drugs: Case Histories. New York, NY: Plenum Press; 1993: 315-350.

3. Carino GP, Mathiowitz E. Review on insulin delivery highlighting several enhancement strategies for oral bioavailability. Adv Drug Deliv Rev. 1999;35:249-257.

4. Wu ZM, Zhou L, Guo XD, et al. HP55-coated capsule containing PLGA/RS nanoparticles for oral delivery of insulin. Int $J$ Pharm. 2012;425:1-8.

5. Zhang Y, Wei W, Lv P, Wang L, Ma G. Preparation and evaluation of alginate-chitosan microspheres for oral delivery of insulin. Eur J Pharm Biopharm. 2011;77:11-19.

6. Makhlof A, Tozuka Y, Takeuchi H. Design and evaluation of novel $\mathrm{pH}-$ sensitive chitosan nanoparticles for oral insulin delivery. Eur J Pharm Sci. 2011;42:445-451.

7. Liechty WB, Caldorera-Moore M, Phillips MA, Schoener C, Peppas NA. Advanced molecular design of biopolymers for transmucosal and intracellular delivery of chemotherapeutic agents and biological therapeutics. J Control Release. 2011;155:119-127.

8. Zhang N, Li J, Jiang W, et al. Effective production and controlled release of insulin by cationic $\beta$-cyclodextrin polymers from alginate/chitosan nanoparticles. Int J Pharm. 2010;393:212-218.

9. Jain S, Rathi VV, Jain M, Das C, Godugu C. Folate-decorated PLGA nanoparticles as a rationally designed vehicle for the oral delivery of insulin. Nanomedicine. 2012;7:1311-1337.
10. Sugihara H, Yamamoto H, Kawashima Y, Takeuchi H. Effectiveness of submicronized chitosan-coated liposomes in oral absorption of indomethacin. J Liposome Res. 2012;22:72-79.

11. Liu J, Gong T, Fu H, et al. Solid lipid nanoparticles for pulmonary delivery of insulin. Int J Pharm. 2008;356:333-344.

12. Damgé C, Michel C, Aprahamian M, Couvreur P. New approach for oral administration of insulin with polyalkylcyanoacrylate nanocapsules as drug carrier. Diabetes. 1988;37:246-251.

13. Florence AT. The oral absorption of micro-and nanoparticulates: neither exceptional nor unusual. Pharm Res. 1997;14:259-266.

14. Blanco MD, Alonso MJ. Development and characterization of proteinloaded poly(lactide-co-glycolide) nanospheres. Eur J Pharm Biopharm. 1997;43:287-294.

15. Parajó Y, d'Angelo I, Horváth A, et al. PLGA: poloxamer blend micro-and nanoparticles as controlled release systems for synthetic proangiogenic factors. Eur J Pharm Sci. 2010;41:644-649.

16. Kumari A, Yadav SK, Yadav SC. Biodegradable polymeric nanoparticle based drug delivery systems. Colloids Surf B Biointerfaces. 2010;75:1-18.

17. Mittal G, Carswell H, Brett R, Currie S, Ravi Kumar MNV. Development and evaluation of polymer nanoparticles for oral delivery of estradiol to rat brain in a model of Alzheimer's pathology. J Control Release. 2011;150:220-228.

18. Sarti F, Perera G, Hintzen F, et al. In vivo evidence of oral vaccination with PLGA nanoparticles containing the immunostimulant monophosphoryl lipid A. Biomaterials. 2011;32:4052-4057.

19. Hosseininasab S, Pashaei-Asl R, Khandaghi AA, Nasrabadi HT, Nejati-Koshki K, Akbarzadeh A, Joo SW, Hanifehpour Y, Davaran S, Synthesis, characterization, and in vitro studies of PLGA-PEG nanoparticles for oral insulin delivery. Chem Biol Drug Des. 2014;84:307-315.

20. Bazille D, Prud'homme C, Bassoullet MT, et al. PEG-PLA nanoparticles avoid uptake by the mononuclear phagocytes system. $J$ Pharm Sci. 1995;84:493-498.

21. Reix N, Parat A, Seyfritz E, et al. In vitro uptake evaluation in Caco-2 cells and in vivo results in diabetic rats of insulin-loaded PLGA nanoparticles. Int J Pharm. 2012;437:213-220.

22. De Campos A, Sanchex A, Gref R, Clavo P, Alonso MJ. The effect of a PEG versus a chitosan coating on the interaction of drug colloidal carriers with the ocular mucosa. Eur J Pharm Sci. 2003;20:73-81.

23. Villa A, Sanchez A, Tobio M, Calvo P, Alonso MJ. Design of biodegradable particles for protein delivery. J Control Release. 2002;78:15-24.

24. Avgoustakis K, Beletsi A, Panagi Z, et al. Effect of copolymer composition on the physicochemical characteristics, in vitro stability, and biodistribution of PLGA-mPEG nanoparticles. Int J Pharm. 2003;259:115-127.

25. Ensign LM, Cone R, Hanes J. Oral drug delivery with polymeric nanoparticles: the gastrointestinal mucus barriers. Adv Drug Delivery Rev. 2012;64:557-570.

26. Mu L, Feng SS. A novel controlled release formulation for the anticancer drug paclitaxel $\left(\right.$ Taxol $\left.^{\mathbb{}}\right)$ : PLGA nanoparticles containing vitamin E TPGS. J Control Release. 2003;86:33-48.

27. DIez FV, Sastre H, Coca J. Effect of the operating conditions on the preparations of stannous octoate from stannous oxide. Ind Eng Chem Res. 1988;27:845.

28. Snedecor GW, Cochran WG. Statistical Method. 9th ed. Ames, IA: Iowa State University Press; 1999.

29. Santander-Ortega MJ, Bastos-González D, Ortega-Vinuesa JL, Alonso MJ. Insulin-loaded PLGA nanoparticles for oral administration: an in vitro physico-chemical characterization. J Biomed Nanotech. 2009;5:45-53.

30. Yang J, Sun H, Song C. Preparation, characterization and in vivo evaluation of $\mathrm{pH}$-sensitive oral insulin-loaded poly(lactic-co-glycolic acid) nanoparticles. Diabetes Obes Metab. 2012;14:358-364.

31. van de Weert M, Hoechstetter J, Hennink WE, Crommelin DJ. The effect of a water/organic solvent interface on the structural stability of lysozyme. J Control Release. 2000;68:351-359.

32. Abdelwahed W, Degobert G, Stainmesse S, Fessi H. Freeze-drying of nanoparticles: formulation, process and storage considerations. $A d v$ Drug Deliv Rev. 2006;58:1688-1713. 
33. Morishita M, Morishita I, Takayama K, Machida Y, Nagai T. Novel oral microspheres of insulin with protease inhibitor protecting from enzymatic degradation. Int J Pharm. 1992;78:1-7.

34. Tobio M, Sanchez A, Vila A, et al. The role of PEG on the stability in digestive fluids and in vivo fate of PEG-PLA nanoparticles following oral administration. Colloid Surf B. 2000;18:315-323.

35. Ruan G, Feng SS, Li QT. Effects of material hydrophobicity on physical properties of polymeric microspheres formed by double emulsion process. J Control Release. 2002;84;151-160.

36. Bendayan M, Ziv E, Gingras D, Ben-Sasson R, Bar-On H, Kirdon M. Biochemical, and morpho-cytochemical evidence for the intestinal absorption of insulin in control and diabetic rats. Comparison between the effectiveness of duodenal and colon mucosa. Diabetologia. 1994;37:119-126.

37. Cui F-D, Tao A-J, Cun D-M, Zhang L-Q, Shi K. Preparation of insulin loaded PLGA-Hp55 nanoparticles for oral delivery. J Pharm Sci. 2007;96:421-427.

38. Pan Y, Li YJ, Gao P, Ding PT, Xu H, Zheng JM. Enhancement of gastrointestinal absorption of chitosan-coated insulin-loaded poly(lactic-coglycolic) acid nanoparticles. Acta Pharm Sinica. 2003;38:467-470.

39. Sun S, Liang N, Kawashima Y, Xia D, Cui F. Hydrophobic ion pairing of an insulin-sodium deoxycholate complex for oral delivery of insulin. Int J Nanomed. 2011;6:3049-3056.

40. Kumar PS, Ramakrishna S, Saini TR, Diwan PV. Influence of microencapsulation method and peptide loading on formulation of poly(lactideco-glycolide) insulin nanoparticles. Pharmaize. 2006;61:613-617.
41. Asad M, Aslam M, Munir TA, Nadeem A. Effect of acacia nilotica leaves extract on hyperglycemia, lipid profile and platelet aggregation in streptozotocin induced diabetic rats. J Ayub Med Coll. 2011;23:3-7.

42. Mwafy SN, Yassin MM. Physiological changes associated with streptozotocin induced experimental diabetic rats. J Al Azhar University-Gaza (ICBAS Special Issue). 2010;12:31-35.

43. Sajitha PC, Punitha D, Danya U, Udhayasankar MR, Baluprakash T, Arumugasamy K. Antidiabetic activity of anisochillusdysophylloides wall. exbenth. (lamiaceae) in streptozotocin induced Type 2 diabetes in male wistar albino rats. IJPAES. 2012;2:40-43.

44. Adaramoye OA. Antidiabetic effect of kolaviron, a biflavonoid complex isolated from Garcinia kola seeds in wistar rats. Afr Health Sci. 2012;12:498-506.

45. Tedong L, Theophile D, Dzeufiet PDD, et al. Antihyperglycemic and renal protective activities of Anacardiumoccidentale leaves in streptozotocin induced diabetic rats. Afr J Trad CAM. 2006;3:23-35.

46. Bolkent S, Bolkent S, Yanardag R, Tunali S. Protective effect of vanadyl sulfate on the pancreas of streptozotocin-induced diabetic rats. Diab Res Clin Prac. 2005;70:103-109.

47. Chen MC, Sonaje K, Chen KJ, Sung HW. A review of the prospects for polymeric nanoparticle platforms in oral insulin delivery. Biomaterials. 2011;32:9826-9838.
International Journal of Nanomedicine

\section{Publish your work in this journal}

The International Journal of Nanomedicine is an international, peerreviewed journal focusing on the application of nanotechnology in diagnostics, therapeutics, and drug delivery systems throughout the biomedical field. This journal is indexed on PubMed Central, MedLine, CAS, SciSearch $\AA$, Current Contents ${ }^{\circledR} /$ Clinical Medicine,

\section{Dovepress}

Journal Citation Reports/Science Edition, EMBase, Scopus and the Elsevier Bibliographic databases. The manuscript management system is completely online and includes a very quick and fair peer-review system, which is all easy to use. Visit http://www.dovepress.com/ testimonials.php to read real quotes from published authors. 